\title{
Inferindo a importância dos atributos do transporte coletivo a partir da satisfação dos usuários
}

\author{
Mariana Müller Barcelos', Luis Antonio Lindau², Brenda Medeiros Pereira ${ }^{3}$, \\ Ângela de Moura Ferreira Danilevicz ${ }^{4}$, Carla Schwengber ten Caten ${ }^{5}$ \\ 1WRI Brasil, mariana.barcelos@wri.org \\ 2WRI Brasil, toni.lindau@wri.org \\ 3WRI Brasil, brenda.medeiros@wri.org \\ ${ }^{4}$ Departamento de Engenharia de Produção e Transportes, UFRGS, angelamfd@producao.ufrgs.br \\ ${ }^{5}$ Departamento de Engenharia de Produção e Transportes, UFRGS, tencaten@producao.ufrgs.br
}

\section{Recebido:}

20 de fevereiro de 2017

Aceito para publicação:

21 de setembro de 2017

Publicado:

29 de outubro de 2017

Editor de área:

Flávio Cunto

\section{Palavras-chaves:}

Transporte coletivo por ônibus, Qualidade com foco no usuário,

Pesquisa de satisfação,

Importância declarada,

Importância derivada.

\section{Keywords:}

Bus public transport,

Quality focused on users,

Satisfaction survey,

Stated importance,

Derived importance.

DOI:10.14295/transportes.v25i3.1336

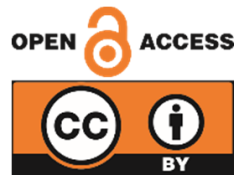

\begin{abstract}
RESUMO
Este estudo tem foco na obtenção da importância dos atributos do transporte coletivo por ônibus. A importância pode ser obtida por duas diferentes abordagens: a importância declarada (na qual os usuários respondem diretamente sobre a importância) e a importância derivada (na qual, a partir das avaliações de satisfação, se infere a importância através de técnicas estatísticas). Com o objetivo de verificar a existência de uma relação entre as duas abordagens que possa tornar dispensável a aplicação de perguntas de importância, conduziu-se entrevistas junto a usuários de ônibus da cidade de Porto Alegre, determinando-se a importância derivada e declarada dos atributos. As análises não demonstraram uma correspondência direta entre as abordagens. No entanto, utilizando um gráfico de dispersão importância versus satisfação, identificou-se uma correspondência entre os atributos localizados no quadrante de alta prioridade. Portanto, foi possível estabelecer uma relação qualitativa entre as abordagens declarada e derivada.
\end{abstract}

\begin{abstract}
This study focuses on obtaining the importance of the attributes of public transportation by bus. The importance can be obtained by two different approaches: the stated importance (in which, the users answer directly about the importance) and the derived importance (in which, from the satisfaction ratings, it is inferred the importance through statistical techniques). In order to verify the existence of a relationship between the two approaches that would make unnecessary the application of importance questions, we conducted interviews with the bus users in Porto Alegre city, determining the derived and stated importance of the attributes. Analyzes have not demonstrated a direct correspondence between the approaches. However, using an importance versus satisfaction scatterplot, we identified a correspondence between attributes located in the high priority quadrant. Thus, it was possible to establish a qualitative relationship between the stated and derived approaches.
\end{abstract}

\section{INTRODUÇÃO}

Cerca de um terço da população brasileira utiliza transporte coletivo para realizar seus deslocamentos diários. Desses, o modo ônibus se destaca como responsável por 86\% das viagens (ANTP, 2016). Apesar disso, entre 2014 e 2015, os sistemas de ônibus perderam três milhões de passageiros diários (NTU, 2016), resultado da falta de competitividade dos ônibus frente a outros modos disponíveis nas cidades. A redução de passageiros impacta diretamente na sustentabilidade econômica dos sistemas, e consequentemente nos valores das tarifas e na qualidade do serviço, o que contribui ainda mais para a redução da atratividade do transporte coletivo por ônibus. 
Diante da necessidade de atrair pessoas para esse modo e manter as que já o utilizam, torna-se essencial investir na qualidade do transporte coletivo por ônibus tendo como foco o usuário. Para entender as necessidades e percepções dos usuários, as pesquisas de satisfação são ferramentas que permitem quantificar e entender se os usuários estão ou não satisfeitos com os aspectos que compõem o sistema de transporte. Tão importante quanto a satisfação atribuída pelos usuários aos diferentes aspectos do sistema, é a importância que cada um desses aspectos tem para o cliente (Matsukuma e Hernandez, 2007). Conhecer a importância dos diferentes atributos, em conjunto com a satisfação, permite identificar e priorizar ações que terão um impacto mais efetivo na melhoria da qualidade para os usuários (Mkpojiogu e Hashim, 2016). Tendo como motivação entender as características mais valorizadas pelos usuários, o presente trabalho tem enfoque na obtenção da importância dos atributos do transporte coletivo por ônibus.

Existem duas formas de se avaliar a importância: através da importância declarada, em que se pergunta diretamente a importância dos atributos do serviço prestado, e através da importância derivada, que é obtida estatisticamente a partir dos resultados da pesquisa de satisfação em relação aos mesmos itens (Chu, 2002). 0 objetivo deste estudo é verificar a existência de uma relação entre a importância declarada e a derivada. Se comprovada esta relação pode ser possível a aplicação de questionários que contenham apenas perguntas de satisfação e, a partir destes resultados, inferir a importância derivada dos atributos do transporte.

Inicialmente é apresentada a fundamentação teórica e a descrição da metodologia adotada para a realização do estudo. Em seguida são apresentadas as análises e os resultados obtidos e, por fim, são apresentadas as considerações finais.

\section{QUALIDADE DO TRANSPORTE COLETIVO POR ÔNIBUS}

Por quase duas décadas, o transporte público urbano teve a qualidade associada apenas a níveis de serviço e a variáveis econômicas, traduzindo apenas os desejos dos operadores do sistema de transporte público e dos órgãos gerenciadores. Foi a partir da década de 1990 que o conceito de qualidade passou a ser utilizado mais amplamente. Desse modo, a qualidade agregou também as visões pela percepção dos usuários, incluindo os desejos e as necessidades da sociedade (Bertozzi e Lima Jr., 1998).

Em diversas cidades do mundo o transporte coletivo por ônibus vem enfrentando a queda de passageiros, resultado da perda de competitividade em relação a outros modos. Apesar disso, estudos mostram que o investimento em um transporte de alta capacidade, integrado à cidade e atendendo às necessidades dos usuários, é capaz de atrair pessoas e reduzir as viagens de veículos privados (Litman, 2017). Além dos benefícios diretos, recebidos pelos usuários do transporte coletivo, a população como um todo se beneficia, uma vez que as externalidades negativas do uso de veículos privados são reduzidas.

De acordo com o Relatório 100 do Transit Cooperative Research Program (Kittelson \& Associates, 2003), a qualidade na área dos transportes é definida pelo “[...] desempenho global medido ou percebido do serviço de transporte do ponto de vista do passageiro". Ferraz e Torres (2004) explicam que a qualidade do transporte público deve ser entendida como instrumento para atrair e manter as pessoas para este modo e que "o passageiro deve ser visto como cliente do sistema e das empresas operadoras, tendo, portanto, direito a um serviço que lhe proporcione satisfação e o motive a continuar utilizando o sistema de transporte público - o modo de transporte mais indicado para as cidades".

O ciclo da qualidade, proposto pela Norma Europeia EN 13816 define a medida de satisfação dos usuários pela diferença entre a qualidade esperada e a percebida do serviço (European Standard, 2002). Uma forma de conhecer a qualidade esperada e a percebida pelos usuários é através de pesquisas junto à população, que permitem a obtenção direta de informações sobre a realidade de quem utiliza o transporte, suas expectativas, interesses e opiniões (Raia Jr. e Moreira, 2001).

Os estudos de satisfação consideram avaliações individuais de determinadas características que compõem o serviço ou produto, os chamados atributos, que possibilitam a identificação pontual de aspectos 
que precisam de melhorias. Conhecer a satisfação em relação a cada atributo permite entender os pontos em que as expectativas dos clientes não estão sendo alcançadas, sendo parte importante na identificação das ações que precisam de melhorias (Matsukuma e Hernandez, 2007).

Como apresentado por diversos autores (Baião et al., 2011; Chu, 2002; Greenland et al., 2016; Gustafsson e Johnson, 2004; Matsukuma e Hernandez, 2007), conhecer apenas a percepção dos clientes em relação aos atributos do serviço muitas vezes não é o suficiente; é também necessário entender o quanto esses atributos influenciam no nível de satisfação geral. Desse modo, a importância relativa entre cada aspecto do serviço possibilita identificar os pontos mais relevantes, de forma que os esforços sejam alocados de maneira eficiente e com o objetivo de obter a satisfação do cliente.

\section{DETERMINAÇÃO DA IMPORTÂNCIA}

A necessidade de conhecer a importância relativa de cada aspecto do serviço torna-se clara uma vez que se sabe que é preciso privilegiar os atributos mais importantes em detrimento dos menos importantes (Matsukuma e Hernandez, 2007). No entanto, não há um consenso claro entre os profissionais sobre a melhor forma de obter a importância e muitos autores mostraram em seus estudos que nem sempre as abordagens fornecem o mesmo resultado (Baião et al., 2011; Greenland et al., 2016; Chu, 2002; Gustafsson e Johnson, 2004; Matsukuma e Hernandez, 2007).

\subsection{Abordagens para determinação da importância}

Em princípio, a importância relativa entre atributos pode ser determinada de duas maneiras distintas: pela importância declarada (também chamada de abordagem direta ou stated-importance) e pela importância derivada (também conhecida por abordagem indireta ou derived-importance). A seguir são apresentadas as duas formas de obtenção da importância e as técnicas geralmente empregadas para sua determinação.

\subsubsection{Importância declarada}

0 método de obtenção da importância declarada é o mais simples e é obtido questionando diretamente o usuário sobre a importância dos atributos. A literatura apresenta diversos métodos para se determinar a importância declarada, como (Matsukuma e Hernandez, 2007): (i) diferencial semântico, em que são definidos dois extremos e o entrevistado marca em uma escala o quão perto dos extremos ele considera a importância de determinado atributo; (ii) escala de ordenamento, na qual o entrevistado ordena os atributos de acordo com a importância que ele considera ter cada um, estabelecendo a importância relativa entre eles; e (iii) soma constante, onde, a partir de uma pontuação total pré-estabelecida, o entrevistado distribui esses pontos entre os atributos considerando a importância relativa de cada um deles.

Cada um dos métodos possui vantagens e limitações e deve ser escolhido de acordo com o tipo de pesquisa, tipo de aplicação e perfil de respondentes. 0 método de diferencial semântico, por exemplo, possui o risco de as pessoas darem alta importância para quase todos os atributos, havendo pouca discriminação entre eles. 0 uso de escala de ordenamento tem a vantagem de forçar a pessoa a priorizar alguns atributos em detrimento de outros, porém o gap entre eles, não é conhecido. Já o método de soma constante, além de forçar a pessoa a estabelecer uma hierarquia entre atributos, permite identificar o quão próxima a importância entre eles foi avaliada. No entanto, dependendo do público entrevistado e da quantidade de atributos, esta pode ser uma forma inapropriada (Matsukuma e Hernandez, 2007).

\subsubsection{Importância derivada}

A importância derivada consiste em, a partir das avaliações de satisfação para as características do transporte, inferir, através de técnicas estatísticas, a importância de cada um dos atributos. Neste método a importância dos atributos é determinada avaliando-se o quanto a satisfação com cada um deles está influenciando na satisfação geral (Baião et al., 2011; Chu, 2002; Gustafsson e Johnson, 2004; Matsukuma e Hernandez, 2007; Souza e Michel, 2007). 
Alguns dos métodos estatísticos empregados para definição da importância derivada, entre outros, são (Gustafsson e Johnson, 2004): (i) regressão múltipla, em que é realizada uma simples regressão de um conjunto de avaliações de atributos em relação a uma variável de interesse dependente; (ii) estimativa pareada normalizada, a qual, assim como a regressão múltipla, utiliza todas as variáveis independentes para explicar a variável dependente, porém ajusta correlações individuais com base na correlação total no modelo; (iii) mínimos quadrados parciais, o qual consiste em um procedimento iterativo de estimativas que integra análise de componentes principais com regressão múltipla, considerando o modelo como uma rede de relações de causa e efeito que contém variáveis latentes; e (iv) regressão de componentes principais, o qual, em sua forma tradicional, analisa a classificação de cada fator separadamente para produzir um conjunto de componentes ou fatores independentes.

A regressão múltipla é a abordagem mais fácil de implementar estatisticamente. 0 principal problema dessa abordagem é a multicolinearidade das variáveis independentes (Gustafsson e Johnson, 2004; Tontini e Silveira, 2005). Os demais métodos removem grande parte dessa multicolinearidade, porém, por introduzir técnicas estatísticas mais complexas e ignorar variáveis correlacionadas, os métodos podem gerar resultados de difícil ou nenhuma interpretação prática (Gustafsson e Johnson, 2004). Este trabalho emprega a regressão linear múltipla, técnica mais simples e usual para definir a importância derivada (Baião et al., 2011). Gustafsson e Johnson (2004) explicam que apesar da dificuldade em se obter um conjunto de variáveis com ausência de multicolinearidade, característica comum desse tipo de dados, é possível aplicar técnicas que reduzem o número de variáveis ou a correlação entre elas, possibilitando o uso do método.

\subsection{Comparação entre importâncias declarada e derivada}

Ao comparar as abordagens da importância declarada e da importância derivada, a literatura aponta para algumas divergências entre ambas. Gustafsson e Johnson (2004), inclusive, afirmam que se pode interpretar de forma diferente a importância encontrada pelos dois métodos.

A importância declarada possui maior validade de face, ou seja, sua validade subjetiva é mais bem aceita uma vez que mede diretamente o que se deseja conhecer (Chu, 2002). Além disso, esta abordagem é bem mais simples, já que não é necessária a utilização de técnicas estatísticas (Baião et al., 2011). No entanto, Souza e Michel (2007) explicam que as pessoas podem ter dificuldade em diferenciar importância de satisfação e, por terem maior familiaridade na avaliação da satisfação, se confundem quando perguntadas sobre importância. A aplicação dos dois tipos de perguntas com a mesma pessoa pode tornar a entrevista cansativa e confusa, pois ele precisa responder duas vezes sobre os mesmos atributos. Além disso, uma das principais desvantagens da importância declarada é a impossibilidade de, na maior parte das vezes, fazer uma diferenciação estatística entre atributos (Chu, 2002).

Por outro lado, Chu (2002) afirma que a abordagem derivada é superior, pois permite que seja calculada a porção da satisfação que é explicada pelo modelo adotado. Além disso, à importância derivada pode ser atribuída a ideia de identificar os atributos que tem maior influencia na satisfação, mas que estão ocultos em sua racionalidade e são avaliados subconscientemente. Este método permite identificar atributos que geram satisfação no cliente mesmo que ele não perceba e em geral está relacionado a atributos primários do serviço, como limpeza e atendimento (Souza e Michel, 2007).

Definir um dos métodos como sendo superior ao outro é difícil, uma vez que cada um apresenta vantagens e desvantagens. Em geral, a importância declarada é mais bem aceita e a importância derivada é tratada com maior cautela já que é obtida indiretamente e em geral não gera resultados idênticos ao método direto (Chu, 2002). De modo geral, a literatura mostra que cada uma das abordagens de importância declarada e derivada tem seus críticos e defensores. No entanto, ambas são relevantes e podem fornecer informações importantes para ajudar os provedores de serviços a melhorar suas ofertas e a satisfação do cliente. Greenland et al. (2016), dizem que, se usadas em conjunto, as duas técnicas se complementam e destacam as dimensões menos e mais tangíveis a serem abordadas. 


\section{METODOLOGIA ADOTADA}

Com o objetivo de verificar a relação entre a importância declarada e a derivada, foram realizadas pesquisas de satisfação e de importância com usuários do transporte coletivo por ônibus na cidade de Porto Alegre. Dos dados de satisfação, determinou-se a importância derivada dos atributos e, dos dados de importância, a importância declarada.

\subsection{Questionário}

O questionário aplicado é composto por quatro módulos:

- Perfil de uso: perguntas sobre a forma como os usuários utilizam o transporte;

- Perfil do cliente: perguntas que caracterizam os usuários;

- Satisfação: perguntas sobre o quanto os usuários estão satisfeitos com quinze atributos do transporte coletivo;

- Importância: importância relativa de quinze atributos do transporte atribuída pelos usuários.

Os quinze atributos considerados no estudo são:

- acesso ao transporte: facilidade de chegar aos pontos de acesso e circular nas estações e terminais;

- disponibilidade: intervalo entre os ônibus, nos horários e locais em que necessito;

- rapidez;

- confiabilidade: chegada no horário previsto;

- facilidade de fazer integrações entre linhas de ônibus e outros modos de transporte, para chegar ao destino;

- conforto dos pontos de ônibus: iluminação, proteção, limpeza, quantidade de pessoas;

- conforto dos terminais de integração: iluminação, proteção, limpeza, quantidade de pessoas;

- conforto dos ônibus: iluminação, limpeza, quantidade de pessoas, assentos;

- atendimento ao cliente: respeito, cordialidade e preparo dos motoristas, cobradores, funcionários e central de atendimento;

- informação ao cliente: sobre linhas, horários e outras informações;

- segurança pública contra roubos, furtos e agressões no caminho e dentro dos ônibus;

- segurança em relação a acidentes de trânsito;

- exposição a ruído e poluição gerados pelos ônibus;

- facilidade em pagar o ônibus e recarregar o cartão de transporte;

- gasto com transporte coletivo por ônibus.

Os módulos de Perfil de uso, Perfil do cliente e Satisfação, bem como os quinze atributos empregados para avaliar satisfação e importância, baseiam-se na Pesquisa de Satisfação Qualiônibus (EMBARQ Brasil, 2014). Apenas o módulo de Importância foi desenvolvido especificamente para este estudo.

O módulo de Satisfação considera a escala de notas da Figura 1, a qual foi utilizada pelos respondentes para avaliar sua satisfação em relação aos quinze atributos e à satisfação geral com o sistema de transporte. Já, para o módulo de importância, foram entregues quinze cartões para os respondentes em ordem aleatória, cada um contendo um dos atributos. Por ser um número grande de atributos, primeiro pediase ao entrevistado que escolhesse os quatro atributos considerados por ele mais importantes, após pedia-se os próximos quatro mais importantes e por fim, os próximos quatro, sobrando apenas três cartões. Em seguida, entre os quatro primeiros cartões selecionados, pedia-se ao respondente que os ordenasse por importância, sendo feito isso também com os outros três grupos selecionados. Foi possível então, criar uma ordem de importância para os quinze atributos avaliados. 


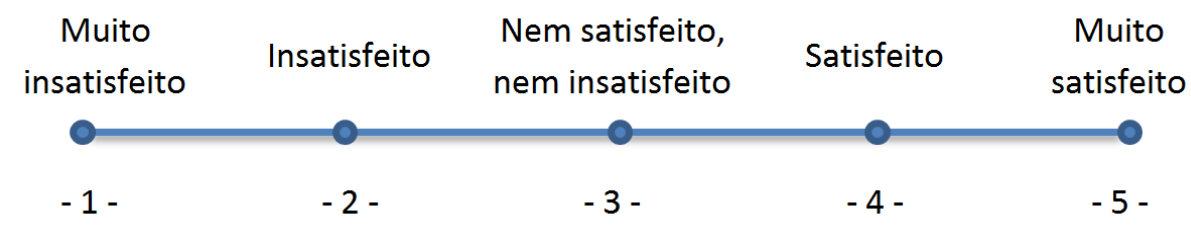

Figura 1: Escala de notas utilizadas no módulo de Satisfação.

\subsection{Cálculo da amostra}

O tamanho da amostra foi determinado por amostragem aleatória simples (Oliveira e Grácio, 2005), conforme Equações 1 e 2. A Equação 1 determina a primeira aproximação da amostra mínima, considerando população desconhecida, nível de confiança fixo de $95 \%$ e variância populacional para o caso de maior heterogeneidade populacional (proporção do evento em estudo é de 0,5). A Equação 2 faz o ajuste da amostra para a população pesquisada. 0 erro amostral adotado é de $8 \%$, devido limitações deste trabalho. Para o tamanho da populacão, utilizou-se a média de passageiros transportados diariamente em 2015 no transporte coletivo por ônibus em Porto Alegre, que, segundo a Empresa Pública de Transporte e Circulacão (EPTC), é de aproximadamente um milhão (EPTC, 2016).

$$
\begin{gathered}
\mathrm{n}_{0}=1 / \mathrm{E}_{0}{ }^{2} \\
\mathrm{n}=\left(\mathrm{N} \cdot \mathrm{n}_{0}\right) /\left(\mathrm{N}+\mathrm{n}_{0}\right)
\end{gathered}
$$

Em que: $\mathrm{n}_{0}$ : primeira aproximação do tamanho mínimo da amostra;
$\mathrm{E}_{0}$ : erro amostral tolerável;
n: tamanho mínimo da amostra;
$\mathrm{N}$ : tamanho da população.

\subsection{Coleta dos dados}

O serviço na cidade é operado por uma empresa pública e três consórcios que se dividem entre quatro bacias na cidade: norte, sul, leste e transversal (EPTC, 2015). A coleta dos dados foi realizada por um pesquisador entre 21 de agosto e 19 de setembro de 2014 com usuários do transporte coletivo por ônibus da cidade de Porto Alegre. As entrevistas foram embarcadas, em que o entrevistador aborda o usuário enquanto ele está realizando sua viagem. Foram realizadas pesquisas apenas em dias úteis, entre $7 \mathrm{~h}$ e 19h, em 12 linhas das quatro empresas, sendo a distribuição das entrevistas proporcional à demanda das quatro empresas operadoras e de forma a abranger os usuários das principais regiões da cidade.

\subsection{Análises}

Após aplicação dos questionários, utilizou-se planilha eletrônica para tabulação dos dados. A seguir são apresentados os métodos de análise adotados.

\subsubsection{Perfil de Uso e do cliente}

Os módulos de Perfil de uso e Perfil do cliente permitem conhecer a amostra pesquisada, além de permitir estratificações para entender melhor a opinião de determinado perfil. Neste trabalho foram construídos gráficos de barras com estes resultados e as análises limitaram-se a avaliar possíveis vieses na amostra.

\subsubsection{Satisfação}

0 módulo de Satisfação gera, para cada respondente, notas de satisfação de 1 a 5 para cada atributo e para a satisfação geral. Para obtenção de uma nota única para cada fator da qualidade e satisfação geral, calcula-se a média aritmética das notas atribuídas por todos os respondentes. 


\subsubsection{Importância declarada}

Da aplicação dos questionários é possível identificar, para cada respondente, uma ordem do $1^{\circ}$ atributo até o 15을 mais importante. A partir dessa ordem, são atribuídos pesos aos atributos definidos pelo inverso de sua posição ( $4^{\circ}$ atributo mais importante recebeu peso $1 / 4=0,25$, por exemplo) (Ribeiro et al., 2001). A partir desses pesos, calcula-se uma média aritmética considerando todos os respondentes e em seguida, essa média é transformada em notas de 0 a 10 para facilitar a comparação com a importância derivada. Os atributos são então ordenados por importância a partir dessas notas.

\subsubsection{Importância derivada}

A importância derivada tem origem nas avaliações de satisfação atribuídas pelos usuários aos atributos do sistema de transporte e na satisfação geral com o serviço prestado. A partir das notas de satisfação é aplicada a técnica da regressão linear múltipla para gerar um modelo que representa um comportamento linear entre a satisfação geral, tratada como variável dependente (Y) e as satisfações em relação aos atributos, tratadas como variáveis independentes (X). No modelo representado na Equação 3 a importância relativa entre atributos é determinada pelos coeficientes das variáveis independentes (coeficientes $\beta$ ): quanto maior este coeficiente, maior é a influência do atributo correspondente sobre a satisfação geral e maior é, portanto, a importância do atributo.

$$
Y=\beta_{0}+\beta_{1} \cdot X_{1}+\beta_{2} \cdot X_{2}+\ldots+\beta_{i} \cdot X_{i}+\ldots+\beta_{n} \cdot X_{n}
$$

Em que: Y: índice da satisfação geral (variável dependente);

$\beta_{\mathrm{i}}$ : índice de importância do atributo i (coeficientes das variáveis independentes);

$\mathrm{X}_{\mathrm{i}}$ : índice da satisfação do atributo i (variáveis independentes);

$\mathrm{n}$ : número de atributos avaliados.

Os dados para regressão foram tratados como dados histórico, podendo existir uma correlação entre as variáveis independentes. Para entender melhor a influência da correlação entre variáveis no coeficiente $\beta$, também foram realizadas análises considerando apenas alguns dos atributos. A partir dos coeficientes $\beta$ obtidos na regressão linear com todos os atributos, calcula-se notas de 0 a 10 para a importância dos atributos para que ambos os métodos, declarado e derivado, estejam na mesma escala, facilitando as análises e comparações.

\subsubsection{Gráfico de dispersão Importância versus Satisfação}

Outra análise realizada foi a que considera importância e satisfação (ou desempenho) combinadas com objetivo de identificar quais atributos do serviço devem ter prioridade e precisam com mais urgência de melhorias. Assim, esta análise permite priorizar os atributos de acordo com a importância relativa entre eles e com a satisfação conferida pelos usuários.

A análise consiste na construção de um gráfico de dispersão, em que o eixo horizontal é a importância e o eixo vertical, a satisfação (Bitencourt et al., 2014). Dessa forma, ficam definidos quatro quadrantes e, dependendo em qual deles o atributo está, se pode classificá-lo em:

- atributos de alta prioridade: corresponde aos atributos que possuem alta importância e baixo desempenho (ou insatisfação por parte dos usuários). São nestes atributos que devem ser concentrados os esforços de melhoria;

- atributos de baixa prioridade: são os atributos de baixa importância e baixo desempenho.

- atributos com desempenho adequado: corresponde aos atributos de alta importância e alta satisfação;

- atributo de vantagem competitiva ou com excesso de desempenho: são os atributos de pouca importância e que têm um bom desempenho.

Considerando as duas abordagens de determinação da importância, direta e indireta, foram construídos gráficos de dispersão representando as notas de satisfação e de importância dos atributos. Para as notas da satisfação são utilizadas as notas de 1 a 5 . A divisão dos quadrantes é localizada na nota 3, ou 
seja, notas acima de 3 representam satisfação e, abaixo, insatisfação. Já, para a importância, dividiramse os atributos entre a metade mais importante e a metade menos importante. Para tanto, como interseção dos eixos calculou-se a mediana, considerando as notas de 0 a 10 de importância.

\section{RESULTADOS E ANÁLISES}

Após coleta dos dados e tabulação, os métodos de análise foram aplicados. A seguir são apresentados os principais resultados.

\subsection{Perfil de Uso e do cliente}

Os resultados dos módulos de Perfil de uso e Perfil do cliente foram coerentes e não indicaram vieses na amostra. Um exemplo claro é o da distribuição dos horários em que os entrevistados utilizam o transporte por ônibus (Figura 2), mesmo não tendo sido feito uma estratificação da amostra por horário de entrevista, foi possível identificar uma distribuição típica de pico manhã, pico tarde e entre pico, mostrando uma coerência com a distribuição de demanda ao longo do dia.

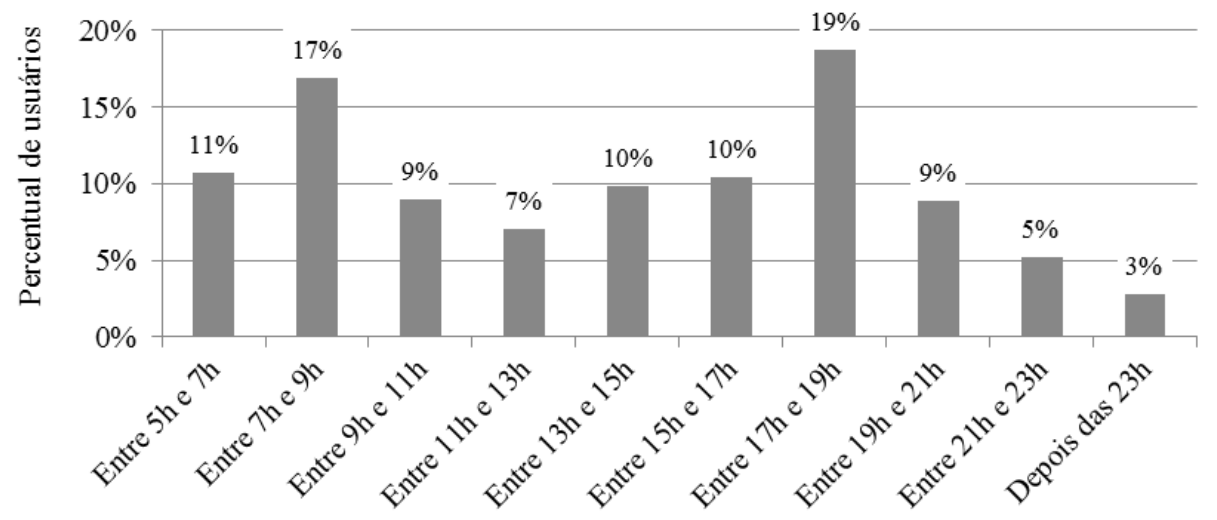

Faixas horárias em que se utiliza o transporte coletivo por ônibus

Figura 2: Distribuição dos horários em que os entrevistados utilizam o transporte por ônibus com maior frequência

\subsection{Satisfação}

As notas médias de satisfação permitem identificar as características do sistema de transporte que possuem maior e menor grau de satisfação por parte dos usuários. Os resultados são apresentados na Tabela 1, onde para cada atributo e para a satisfação geral, é associada uma nota de 1 a 5.

Tabela 1: Notas de Satisfação dos usuários do transporte por ônibus de Porto Alegre

\begin{tabular}{lr}
\hline Atributos & Notas 1-5 \\
\hline Pagamento & 3,918 \\
Atendimento & 3,571 \\
Acesso & 3,559 \\
Informação & 3,551 \\
Integração & 3,442 \\
Segurança acidentes de trânsito & 2,985 \\
Rapidez & 2,913 \\
Disponibilidade & 2,857 \\
Gasto & 2,683 \\
\hline
\end{tabular}


Tabela 1: Notas de Satisfação dos usuários do transporte por ônibus de Porto Alegre (continuação)

\begin{tabular}{lr}
\hline Atributos & Notas 1-5 \\
\hline Confiabilidade & 2,634 \\
Conforto dos terminais & 2,582 \\
Conforto dos ônibus & 2,528 \\
Conforto dos pontos de ônibus & 2,130 \\
Ruído e poluição & 2,099 \\
Segurança pública & 2,085 \\
Satisfação Geral & $\mathbf{3 , 0 3 7}$ \\
\hline
\end{tabular}

\subsection{Importância declarada}

A partir dos resultados do módulo de Importância e da atribuição de pesos para a ordem em que os atributos foram alocados, obteve-se uma ordem de atributos por importância e as respectivas notas de 0 a 10. Os resultados são apresentados na Tabela 2.

Tabela 2: Ordem dos atributos pela importância declarada

\begin{tabular}{llll}
\hline Ordem dos atributos & Média dos pesos & Nota 0-10 \\
1 & Segurança pública & 0,519 & 10,0 \\
2 & Rapidez & 0,326 & 5,1 \\
3 & Disponibilidade & 0,303 & 4,6 \\
4 & Segurança acidentes de trânsito & 0,256 & 3,4 \\
5 & Conforto dos ônibus & 0,227 & 2,7 \\
6 & Acesso & 0,218 & 2,4 \\
7 & Gasto & 0,207 & 2,1 \\
8 & Confiabilidade & 0,206 & 2,1 \\
9 & Conforto dos pontos de ônibus & 0,184 & 1,6 \\
10 & Ruído e poluição & 0,174 & 1,3 \\
11 & Informação & 0,161 & 1,0 \\
12 & Atendimento & 0,150 & 0,7 \\
13 & Conforto dos terminais & 0,136 & 0,4 \\
14 & Pagamento & 0,132 & 0,3 \\
15 & Integração & 0,121 & 0,0 \\
\hline
\end{tabular}

\subsection{Importância derivada}

A partir das notas de satisfação foi aplicada a técnica da regressão linear múltipla, gerando um coeficiente $\beta$ que corresponde ao grau de influencia que cada atributo tem sobre a satisfação geral, ou seja, a importância do atributo. Ao aplicar a regressão considerando as notas de satisfação dos quinze atributos, chegou-se a um modelo em que grande quantidade das variáveis não é significativa (considerando nível de significância de 5\% e intervalo de confiança de 95\%). Foram então realizadas regressões com apenas alguns dos atributos a fim de tentar entender melhor o comportamento das variáveis. A Tabela 3 apesenta um resumo dos valores de $R^{2}$, coeficiente $\beta$ e valor- $p$ obtidos para as regressões que consideraram todos os atributos e apenas os 4, 5, 6 e 7 primeiros atributos encontrados na regressão com todos os atributos. 
Tabela 3: Resumo dos principais resultados associados às regressões lineares realizadas.

\begin{tabular}{|c|c|c|c|c|c|c|c|c|c|c|}
\hline \multicolumn{11}{|l|}{ Estatística de regressão } \\
\hline & \multicolumn{2}{|c|}{ Todos os atributos } & \multicolumn{2}{|c|}{4 atributos } & \multicolumn{2}{|c|}{5 atributos } & \multicolumn{2}{|c|}{6 atributos } & \multicolumn{2}{|c|}{7 atributos } \\
\hline R-Quadrado & \multicolumn{2}{|c|}{0,558} & \multicolumn{2}{|c|}{0,521} & \multicolumn{2}{|c|}{0,534} & \multicolumn{2}{|c|}{0,544} & \multicolumn{2}{|c|}{0,549} \\
\hline \multirow[t]{2}{*}{ Observações } & \multicolumn{2}{|c|}{161} & \multicolumn{2}{|c|}{161} & \multicolumn{2}{|c|}{161} & \multicolumn{2}{|c|}{161} & \multicolumn{2}{|c|}{161} \\
\hline & $\begin{array}{c}\text { Coefic. } \\
\beta\end{array}$ & Valor-p & $\begin{array}{l}\text { Coe- } \\
\text { fic. } \beta\end{array}$ & $\begin{array}{c}\text { Valor- } \\
\mathrm{p}\end{array}$ & $\begin{array}{c}\text { Coefic. } \\
\beta\end{array}$ & $\begin{array}{c}\text { Valor- } \\
\mathrm{p}\end{array}$ & $\begin{array}{c}\text { Coefic. } \\
\beta\end{array}$ & $\begin{array}{c}\text { Valor- } \\
\mathrm{p}\end{array}$ & $\begin{array}{c}\text { Coefic. } \\
\beta\end{array}$ & $\begin{array}{c}\text { Valor- } \\
\mathrm{p}\end{array}$ \\
\hline Interseção & $-0,181$ & 0,660 & 0,576 & 0,004 & 0,274 & 0,267 & $-0,021$ & 0,943 & $-0,092$ & 0,756 \\
\hline Conforto dos ônibus & 0,285 & 0,000 & 0,343 & 0,000 & 0,316 & 0,000 & 0,310 & 0,000 & 0,305 & 0,000 \\
\hline Disponibilidade & 0,228 & 0,000 & 0,294 & 0,000 & 0,290 & 0,000 & 0,262 & 0,000 & 0,248 & 0,000 \\
\hline Segurança pública & 0,140 & 0,032 & 0,169 & 0,004 & 0,179 & 0,002 & 0,170 & 0,004 & 0,155 & 0,009 \\
\hline Gasto & 0,137 & 0,015 & 0,150 & 0,003 & 0,132 & 0,010 & 0,124 & 0,015 & 0,118 & 0,020 \\
\hline Informação & 0,103 & 0,090 & - & - & 0,115 & 0,045 & 0,116 & 0,042 & 0,108 & 0,059 \\
\hline Acesso & 0,100 & 0,154 & - & - & - & - & 0,119 & 0,067 & 0,114 & 0,081 \\
\hline Rapidez & 0,065 & 0,307 & - & - & - & - & - & - & 0,076 & 0,190 \\
\hline $\begin{array}{l}\text { Segurança acidentes de trân- } \\
\text { sito }\end{array}$ & 0,057 & 0,366 & - & - & - & - & - & - & - & - \\
\hline Confiabilidade & 0,054 & 0,427 & - & - & - & - & - & - & - & - \\
\hline Integração & 0,031 & 0,671 & - & - & - & - & - & - & - & - \\
\hline Atendimento & 0,027 & 0,629 & - & - & - & - & - & - & - & - \\
\hline Ruído e poluição & 0,026 & 0,719 & - & - & - & - & - & - & - & - \\
\hline Conforto dos terminais & $-0,009$ & 0,938 & - & - & - & - & - & - & - & - \\
\hline Pagamento & $-0,040$ & 0,614 & - & - & - & - & - & - & - & - \\
\hline $\begin{array}{l}\text { Conforto dos pontos de ôni- } \\
\text { bus }\end{array}$ & $-0,060$ & 0,381 & - & - & - & - & - & - & - & - \\
\hline
\end{tabular}

Tabela 4: Ordem dos atributos pela importância derivada

\begin{tabular}{|c|c|c|c|}
\hline \multicolumn{4}{|c|}{ IMPORTÂNCIA DERIVADA } \\
\hline \multicolumn{2}{|c|}{ Ordem dos atributos } & Coeficiente $\beta$ & Nota $0-10$ \\
\hline 1 & Conforto dos ônibus & 0,285 & 10,0 \\
\hline 2 & Disponibilidade & 0,229 & 8,4 \\
\hline 3 & Segurança pública & 0,140 & 5,8 \\
\hline 4 & Gasto & 0,137 & 5,7 \\
\hline 5 & Informação & 0,104 & 4,8 \\
\hline 6 & Acesso & 0,099 & 4,6 \\
\hline 7 & Rapidez & 0,063 & 3,6 \\
\hline 8 & Segurança acidentes de trânsito & 0,056 & 3,4 \\
\hline 9 & Confiabilidade & 0,054 & 3,3 \\
\hline 10 & Integração & 0,032 & 2,7 \\
\hline 11 & Atendimento & 0,031 & 2,7 \\
\hline 12 & Ruído e poluição & 0,026 & 2,5 \\
\hline 13 & Conforto dos terminais & $-0,006$ & 1,6 \\
\hline 14 & Pagamento & $-0,040$ & 0,6 \\
\hline 15 & Conforto dos pontos de ônibus & $-0,062$ & 0,0 \\
\hline
\end{tabular}

Com base nos resultados das regressões, percebe-se que a ordem dos atributos mais importantes (avaliada pelo coeficiente $\beta$ ) se mantém a mesma, independente da quantidade de atributos nos diferentes modelos de regressão. Além disso, nota-se que quanto maior a importância do atributo, menor é o valor-p correspondente. Pode-se inferir, portanto, que, para os atributos mais importantes, a técnica da regressão linear cumpre os objetivos de estabelecer uma ordem entre eles. Como o objetivo do uso da técnica neste estudo não é construir o modelo em si, mas sim encontrar uma relação entre a importância 
dos atributos, considerou-se adequada a utilização da ordem resultante da regressão linear utilizando todos os atributos. Após, calculou-se as notas de 0 a 10 para a importância derivada dos atributos, conforme apresentado na Tabela 4.

\subsection{Comparação do ordenamento dos atributos pelas abordagens declarada e derivada}

Com base nos dois métodos para determinação da importância relativa entre atributos consideradas neste trabalho, obteve-se uma ordem de atributos por importância e as respectivas notas para cada uma das abordagens (Tabelas 2 e 4). A comparação dos atributos quanto ao ordenamento pela importância demostra que os dois métodos não produzem resultados correspondentes, pois existem diversos atributos que se encontram em posições bem diferentes pelas duas abordagens. Considerando as notas, também se verificam várias divergências. Alguns atributos, mesmo estando em posições parecidas nas duas abordagens, como é o caso da Disponibilidade, apresentam notas bastante distintas. Já outros possuem notas próximas, mas encontram-se em diferentes posições, como o atributo Segurança contra acidentes de trânsito.

\subsection{Gráfico de dispersão Importância versus Satisfação}

Com base nos resultados de satisfação, importância declarada e importância derivada foram construídos dois gráficos: Importância declarada versus Satisfação e Importância derivada versus Satisfação , conforme apresentado na Figura 3. Os gráficos permitem avaliar quais são os atributos considerados de alta prioridade pelas duas abordagens de determinação da importância estudadas.

Analisando os resultados obtidos pela análise da importância e satisfação, nota-se claramente que os atributos, em geral, localizam-se nos mesmos quadrantes nas duas abordagens. Atentando para os atributos classificados como de alta prioridade, pode-se identificar uma correspondência entre os itens localizados no quadrante, ou seja, tanto a importância declarada quanto a derivada convergem para a mesma classificação de atributos que carecem de melhorias com maior urgência. Portanto, por esta análise, podem ser identificados sete atributos que possuem alta prioridade, sendo eles: Disponibilidade, Rapidez, Confiabilidade, Conforto dos ônibus, Segurança pública, Segurança contra acidentes de trânsito e Gasto.

Importância DECLARADA versus Satisfação

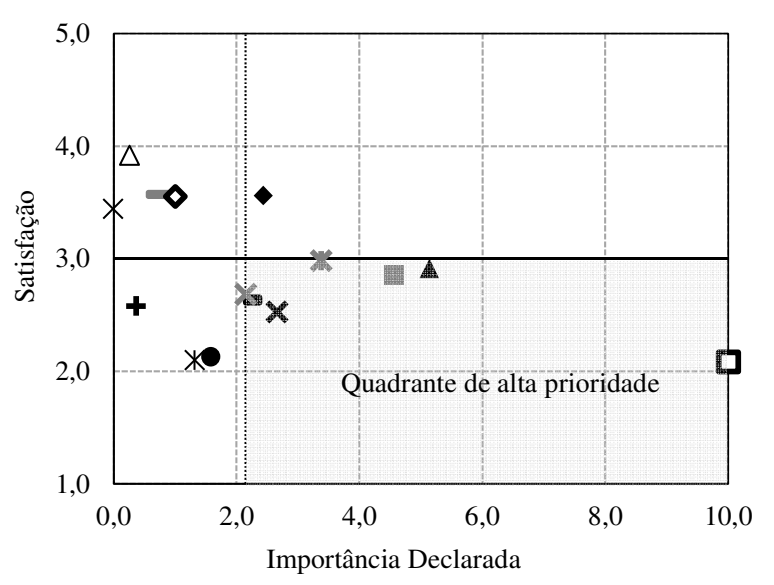

Importância DERIVADA versus Satisfação

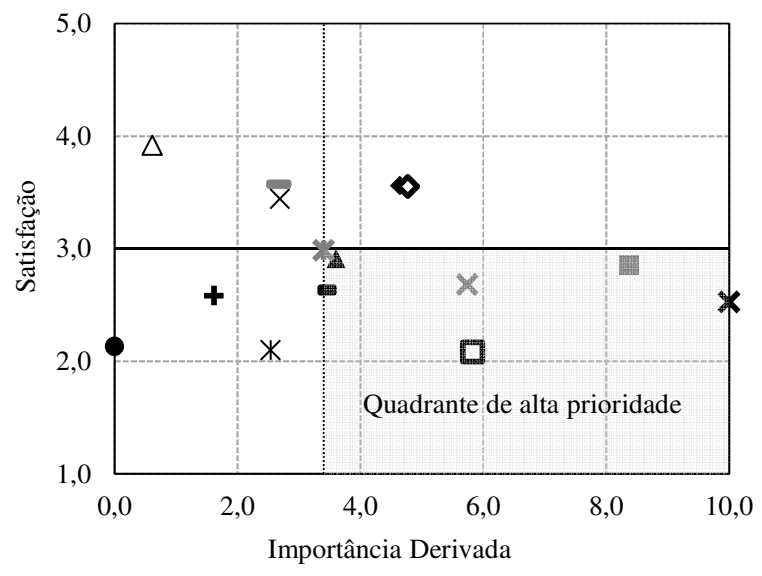

$\begin{array}{lll}\text { • Acesso } & \text { Disponibilidade } & \Delta \text { Rapidez } \\ \text { ×Integração } & \text { ๑Conforto dos pontos de ônibus } & \text { +Conforto dos terminais } \\ \text { - Atendimento } & \bullet \text { Informação } & \square \text { Segurança pública } \\ \text { *Ruído e poluição } & \triangle \text { Pagamento } & \text { XGasto }\end{array}$

- Confiabilidade

XConforto dos ônibus

« Segurança acidentes de trânsito

Figura 3: Gráficos de dispersão Importância declarada versus Satisfação e Importância derivada versus Satisfação 


\section{CONSIDERAÇÕES FINAIS}

Tendo como objetivo a comparação entre as abordagens da importância declarada e importância derivada aplicadas aos atributos do transporte coletivo por ônibus, conclui-se que não há evidências para a existência de uma relação quantitativa e direta entre os dois métodos. Realizando uma análise qualitativa, no entanto, foi possível obter uma relação entre as abordagens, uma vez que ambos os métodos levaram à mesma classificação de atributos de alta prioridade na melhoria da qualidade.

A partir da ordem de atributos obtida por cada um dos métodos, verifica-se que possivelmente as abordagens avaliam aspectos diferentes da importância, como de fato outros trabalhos já haviam apontado. Os atributos Segurança pública e Conforto dos ônibus que foram definidos como os mais importantes pela importância declarada e pela derivada, respectivamente, podem ser exemplos disso. Segurança pública, na avaliação do usuário, provavelmente não é considerada um atributo apenas do transporte, mas do cotidiano, possuindo um peso muito maior que o de outros porque transcende as características do sistema, o que é captado pelo método da importância declarada. Já a importância derivada tende a conferir como atributos mais importantes aqueles que possuem uma avaliação de satisfação mais parecida com a satisfação geral. Isto é, a importância derivada captura os atributos mais relacionados com a imagem do sistema. Isto de fato ocorreu, ao se identificar Conforto do ônibus, um dos atributos que está mais relacionado à imagem do sistema, como o mais importante por este método.

Embora as duas abordagens possam avaliar aspectos diferentes, foi possível obter uma relação qualitativa entre os métodos. Os resultados pela análise do gráfico de dispersão importância versus satisfação permitem identificar os itens que precisam ser prontamente melhorados, tendo os dois métodos levado à mesma indicação de atributos que devem ser priorizados. Logo, para os dados analisados, conclui-se que a importância derivada é uma ferramenta que pode auxiliar na tomada de decisão, já que possibilita identificar atributos prioritários.

Importante observar que o gráfico de dispersão importância versus satisfação não resulta em correspondência de posição quando observado apenas o quadrante de alta prioridade, reforçando a ideia de que a correspondência é qualitativa e não quantitativa, não sendo possível estabelecer uma diferenciação entre atributos dentro do grupo. No caso estudado, sete atributos são considerados de alta prioridade (Disponibilidade, Rapidez, Confiabilidade, Conforto dos ônibus, Segurança pública, Segurança contra acidentes de trânsito e Gasto), o que não possibilita a identificação de aspectos pontuais para definição de ações prioritárias, mas que já torna possível a seleção de um grupo menor de atributos a serem melhorados entre todos que compõem o sistema de transporte.

Para futuras pesquisas, considera-se interessante uma aplicação de formulários mais ampla, que abranja outras localidades e amostra com maior representatividade estatística. As mesmas análises realizadas neste trabalho também poderiam considerar outras técnicas, além da regressão linear múltipla para obtenção da importância derivada, bem como outras análises qualitativas, além do gráfico de dispersão importância versus desempenho.

\section{REFERÊNCIAS BIBLIOGRÁFICAS}

ANTP, 2016 - Associação Nacional dos Transportes Públicos. Sistema de Informações da Mobilidade Urbana da ANTP - Relatório Comparativo 2003/2014. São Paulo/SP, Brasil. Disponível em: <http://files.antp.org.br/2016/9/3/sistemasinformacao-mobilidade--comparativo-2003_2014.pdf> Acesso em: 12 de set 2017.

Baião, A. L.; J. L. Valadares e L. P. Claudino (2011) A importância dos atributos na qualidade percebida em serviços: um estudo comparativo entre os métodos da importância declarada e derivada. Anais do XXXV Encontro da Associação Nacional de Pós-Graduação e Pesquisa em Administração, EnANPAD, Rio de Janeiro, p. 1-16.

Bertozzi, P. P. e O. F. Lima Jr. (1998) A qualidade no serviço de transporte público sob as óticas do usuário, do operador e do órgão gestor. Revista dos Transporte Públicos, n. 081, p. 53-66. Disponível em: <http://www.antp.org.br/_5dotSystem/download/dcmDocument/2013/01/10/BE43F873-30DD-4D62-8997-67F61D0D4FFB.pdf> Acesso em: 19 de jun 2015.

Bitencourt, G. R., A. M. F. Danilevicz e F. D. Michel (2014) Análise de gaps da qualidade nos serviços prestados por operador logístico. Anais da XIV Semana de la Produción Sudamericana, SEPROSUL, Mendoza, v. 1, p. 1-11. 
Chu, R. (2002) Stated-importance versus derived-importance customer satisfaction measurement. Journal of Services Marketing, v. 16, n. 4, p. 285-301. Disponível em: <http://www.emeraldinsight.com/doi/pdfplus/10.

1108/08876040210433202> Acesso em: 19 de jun 2015. DOI: 10.1108/08876040210433202

EMBARQ Brasil (2014) Qualiônibus pesquisa de Satisfação. EMBARQ Brasil, Porto Alegre. Disponível em: <http://d.pr/f/1juZg> Acesso em: 26 de out. 2017.

EPTC (2016) Transporte em números: indicadores anuais do transporte público - Modal ônibus. Empresa Pública de Transporte e Circulação de Porto Alegre. Disponível em: <http://lproweb.procempa.com.br/pmpa/prefpoa/eptc/usu_doc/capitulo_onibus_17jan2016.pdf> Acesso em: 26 de out. 2017.

European Standard (2002) EN 13816: transportation - logistics and services - public passenger transport - service quality definition, targeting and measurement. Bruxelas.

Ferraz, A. C. P.; Torres, I. G. E. Transporte Público Urbano. 2. ed. ampl. e atual. São Carlos.

Greenland, S. J.; I. A. Combe e A. M. Farrell (2016) Stakeholder preference and stated vs derived importance satisfaction research. International Journal of Market Research, vol. 58, n. 1, p.35-56. DOI: 10.2501/IJMR-2016-005

Gustafsson, A. e M. D. Johnson (2004) Determining Attribute Importance in a Service Satisfaction Model. Journal of Service Research. v. 7, n. 2, p. 124-141. DOI: 10.1177/1094670504268453

Kittelson \& Associates, Inc. (2003) Transit Capacity and Quality of Service Manual. Transit Cooperative Research Program: Report 100 (2nd ed.). Washington, DC, USA

Litman, T (2017) Evaluating Public Transit Criticism: systematic analysis of political attacks on high quality transit, and how transportation professionals can effectively respond. Victoria Transport Policy Institute. Disponível em: <http://vtpi.org/railcrit.pdf> Acesso em: 12 de set 2017.

Matsukuma, C. e J. M. da C. Hernandez (2007) Escalas e métodos de análise em pesquisa de satisfação de clientes. Revista de Negócios, v. 12, n. 2, p. 85-103. DOI: 10.7867/1980-4431.2006v11n1p\%p

Mkpojiogu, E. O. C. e N. L. Hashim (2016) Understanding the relationship between Kano model's customer satisfaction scores and self-stated requirements importance. Springer Plus, vol. 5, n. 1. DOI: 10.1186/s40064-016-1860-y

NTU (2016) - Associação Nacional de Empresas de Transportes Urbanos. Ônibus perde 3 milhões de passageiros por dia no Brasil. Revista NTUurbano. Ano IV, número 23. Brasília, 2016. Disponível em: <http://www.ntu.org.br/novo/upload/Publicacao/Pub636120575837109247.pdf> Acesso em: 12 de set 2017.

Oliveira E. F. T. da e M. C. C. Grácio (2005) Análise a respeito do tamanho de amostras aleatórias simples: uma aplicação na área de Ciência da Informação. Revista de Ciência da Informação, v. 6, n. 3. Disponível em: <http://www.brapci.ufpr.br/brapci/v/a/7551> Acesso em 20 de set. 2017.

Raia Junior, A. A. e F. L. Moreira (2001) A Qualidade do Sistema de Transporte Coletivo por Ônibus na Cidade de São Carlos Sob a Ótica dos Usuários. 13º Congresso Brasileiro de Transporte e Trânsito, ANTP, Porto Alegre. Disponível em: <http://www.antp.org.br/_5dotSystem/download/dcmDocument/2013/01/21/4AB735F7-690C-437B-BF15DC4D1A50E477.pdf > Acesso em: 19 de jun 2015.

Ribeiro, J. L. D.; M. E. Echeveste e A. M. F. Danilevicz (2001) A utilização do QFD na otimização de produtos, processos e serviços. Série Monográfica Qualidade, Universidade Federal do Rio Grande do Sul. Fundação Empresa Escola de Engenharia - FEENG, Porto Alegre.

Souza, F. B. B. e F. D. Michel (2007) Avaliação segmentada do sistema de transporte público: uma análise qualitativa para tomada de decisão. Anais do XXI Congresso de Pesquisa e Ensino em Transportes, ANPET, Rio de Janeiro.

Tontini, G. e A. Silveira, (2005) Identification of critical attributes of success in products and services: an alternative to importance - performance analysis. Proceedings of Balas Annual Conference, Madrid: Instituto de Empresa, p. 1-20. 